\title{
COMPARATIVE EVALUATION OF CHRONIC RHINOSINUSITIS PATIENTS BY CT AND NASAL ENDOSCOPIC EXAMINATION
}

\author{
Vishal Bhatnagar1, J. S. Matta ${ }^{2}$ \\ ${ }^{1}$ Assistant Professor, Department of Otorhinolaryngology, RNT Medical College, Udaipur. \\ 2Professor and HOD, Department of Otorhinolaryngology, American International Institute of Medical Sciences, Udaipur.
}

\begin{abstract}
\section{BACKGROUND}

Chronic rhinosinusitis is one of the most commonly encountered diseases of ENT practice. It is characterised by inflammation of mucosa of nasal cavity and sinuses which does not respond to routine treatment up to 12 weeks. It is an extremely common condition affecting $15 \%$ population. It has been seen that the inflammatory sinus disease involves the maxillary and frontal sinuses with the underlying seat of pathology in the tissues of anterior ethmoids. Detailed definition of the syndrome was developed by rhinosinusitis task force of the American Academy of Otolaryngology - Head \& Neck Surgery. For the diagnosis of chronic rhinosinusitis, patients were evaluated by anterior rhinoscopy, nasal endoscopy and CT paranasal sinus especially coronal cuts were preferred. After the development of nasal endoscopy, diagnosis of the nose and paranasal sinuses diseases were made easy by direct examination of nasal cavity and opening of paranasal sinuses as an OPD procedure. For defining the anatomical boundaries and any abnormality in the sinuses frame work CT scan has proven to be beneficial.

The aim of the study was to diagnose chronic rhinosinusitis either by CT scan or endoscopic examination of nasal cavity and paranasal sinuses vis-a-vis is better. Nasal endoscopy has dramatically reduced morbidity and can avoid unnecessary radiation exposure, cost and time in Indian scenario as we have to deal with the poor people in daily practice.
\end{abstract}

\section{MATERIALS AND METHODS}

We evaluated 60 patients of age groups 10 years and above of chronic rhinosinusitis for 3 months or more by nasal endoscopy and CT scan of paranasal sinuses to know the value of both modalities and whether by avoiding CT scan we can avoid unnecessary radiation exposure, cost and time of investigations.

\section{RESULTS}

We had good results by both endoscopy and CT scanning of the patients of chronic rhinosinusitis in assessing the disease as perceived by other studies. The results of endoscopy and CT comparison among 60 patients indicated that for most of the findings there was almost perfect to substantial level of agreement between the two modalities.

\section{CONCLUSION}

Nasal endoscopy is also a good tool for direct visualisation and comparison of sinonasal disease and being an outpatient procedure may reduce unnecessary diagnostic CT scanning. It gives good vision with brilliant illumination of nose and paranasal sinuses. Thus, CT scanning should be reserved for refractory cases not responding to treatment and thereby reducing cost and radiation exposure.

\section{KEYWORDS}

Nasal Endoscopy, Chronic Rhinosinusitis (CRS), CT Scan PNS.

HOW TO CITE THIS ARTICLE: Bhatnagar V, Matta JS. Comparative evaluation of chronic rhinosinusitis patients by CT and nasal endoscopic examination.J.EvolutionMed.Dent.Sci.2017;6(25):2083-2086,DOI:10.14260/Jemds/2017/453

\section{BACKGROUND \\ Chronic rhinosinusitis is one of the most commonly encountered disease in clinical practice. It is characterised by inflammation of the mucosa of nasal cavity and paranasal sinuses for at least 2 months (Lund \& Kennedy, 1995). The lining mucosa of nasal cavity and paranasal sinuses is in continuity, therefore it is rare for the inflammation to affect one without the other and hence rhinosinusitis.[1,2,3]}

Financial or Other, Competing Interest: None

Submission 16-02-2017, Peer Review 14-03-2017,

Acceptance 20-03-2017, Published 27-03-2017.

Corresponding Author:

Dr.J. S. Matta,

Department of Otorhinolaryngology,

American International Institute of

Medical Sciences, Udaipur.

E-mail: drjsmatta@gmail.com

DOI:10.14260/jemds $/ 2017 / 453$

(c) $($ ) $\$$
In the past, diagnosis of sinusitis was based on clinical signs and symptoms of the patient, illumination tests in dark and conventional skiagrams of paranasal sinuses but these tests had several limitations.

For reaching towards a proper diagnosis and management of chronic rhinosinusitis, detailed definition of symptom was developed by the rhinosinusitis task force of the American Academy of Otolaryngology - Head \& Neck Surgery in 1997, consisting of major and minor criteria for diagnosis. [4,5]

The CT scan introduced by Sir Godfrey Hounsfield (1972) and its application in CRS has revolutionised the diagnosis by giving invaluable information. The CT scan has excellent capacity for displaying soft tissue and bones with anatomic variants and mucosal abnormalities.[6] The endoscopy evaluation of nose and paranasal sinuses yields an excellent and accurate diagnostic evaluation of chronic inflammatory rhinonasal pathologies and to add up endoscopy has dramatically reduced morbidity, being on outpatient 
procedure and improved the surgical outcome of resistant cases.[7,8]

Although chronic rhinosinusitis is a clinical \& symptom based diagnosis, it is necessary to perform further investigations as Stankiewicz and Chow showed that only $47 \%$ of patients who were diagnosed with CRS had disease in sinuses on CT, thus leading them to re-evaluate the symptom based definition. ${ }^{[9,10]}$

As it is known that the major drainage pathways located in (OMC) ostiomeatal complex are the root cause of inflammatory sinonasal diseases.[11] Messerklinger (1967), Kennedy et al (1985),[4] Stammberger (1986), Stammberger \& Wolf (1988) suggested seat of pathology is in the middle meatus and anatomic variations in the middle meatus like concha bullosa, paradoxical middle turbinate, bent uncinate process etc. all contributed significantly in chronic rhinosinusitis pathology. With the advent of CT scan and nasal endoscopy, there has been tremendous change in understanding of ostiomeatal complex area and making diagnosis of diseases involving this region. ${ }^{[12,13,14]}$

CT scan and endoscopy provide the ability to accurately scan these areas for evidence of localised disease. Thus, meticulous endoscopic evaluation of the patient must be done in all cases of CRS and if required to reach certain diagnosis, CT scan should also be added limiting unnecessary radiation exposure and costs.

The present study was designed to evaluate the importance and role played by modalities like endoscopy and CT scan in assessment of patients suffering from chronic inflammatory lesions of nose and paranasal sinuses and if we can reserve the CT scanning for patients not responding to the treatment and where we want to see the bony boundaries of sinuses, any bony lesions, dental pathologies (e.g. dental and dentigerous cyst, etc.) and diseases only limited to sinuses not in the nasal cavities, thus to prevent unnecessary radiation exposure and costs to the poor patients.

\section{MATERIALS AND METHODS}

The study was carried out in Department of Otorhinolaryngology, R.N.T. Medical College, Udaipur for 1 year.

A total of 60 patients in the age group 10 years and above irrespective of sex suffering from various sinonasal lesions with or without polyposis for 3 months or more were evaluated by complete history, clinical evaluation, routine investigations, anterior-rhinoscopy, CT scanning and nasal endoscopy.

Patients having malignant neoplasms, congenital abnormality, previous nasal surgery and trauma were excluded from the study.

Approval of the study was obtained from PG review committee of R.N.T. Medical College, Udaipur.

In the study, nasal endoscopy was done using $4 \mathrm{~mm}$ Hopkins rigid nasal endoscopy $0^{\circ}$ and $30^{\circ}$. After explaining the procedure to patients, proper preparation of the nasal cavity and anaesthetisation of nasal cavity was done by xylocaine $4 \%$ with adrenaline cottonoids using standard three pass technique. As the age group we studied was 10 years and above, in some cases endoscopy could visualise adenoids in nasopharynx too. In all the patients of the study after giving full course of antibiotic, antihistaminic and nasal decongestants topically, CT scan was done with preference to coronal cuts as they are identical to the view with endoscopy. Only 60 patients were evaluated by us because we are working in a peripheral medical college which caters to villagers who are from lower socioeconomic group and who cannot afford costly technique and treatment and furthermore it is very difficult to convince them for routine CT scan. Only 60 patients agreed for CT for this study. We cannot increase the no. of patients at present only for diagnosis where we have other good methods for it.

\section{Statistical Method}

Statistical analysis for the data collection forms were tabulated in a Microsoft Excel spreadsheet. Data were then exported to SPSS, version 16.0 for statistical analysis. Chi square and Student's t tests were used for statistical analysis. $P$ value $<0.05$ was considered statistically significant.

\section{RESULTS}

As revealed by Table 1, the various cases in \% of groups in descending order were group 31-40 (33.3\%), 21-30 (26.6\%) with least in group 51-60 (6.6\%). Thus extremes of age group had least number of cases.

Out of 60 patients, 40 were male and 20 female - M: F ratio (2:1) showing male predominance as shown in Figure 1. The most common symptom was nasal obstruction $(83.3 \%)$ followed by nasal discharge (73.3\%), sneezing (70\%), headache $(56.6 \%)$, smell disturbance $(36.6 \%)$ and postnasal drip (20\%). (Table - II).

CT and endoscopic evaluation of the sinuses showed (73.3\%) involvement of anterior ethmoid followed by maxillary sinus (66.6\%), post ethmoid (30\%), frontal sinus (23.3\%) and sphenoid sinus in (16.6\%). (Table - III)

As revealed from the figure 2 the endoscopy could visualize DNS in 53.3\%, inferior turbinate hypertrophy in $33.3 \%$, middle turbinate hypertrophy in $13.3 \%$ and polypoidal mucosa in middle meatus $43.3 \%$. The CT scan showed DNS (60\%), inferior turbinate hypertrophy $(26.6 \%)$, middle turbinate hypertrophy $(13.3 \%)$ and polypoidal mucosa in middle meatus (43.3\%). The DNS was observed by CT scan (60\%) as compared to endoscopy (53.3\%), but the \% of cases of middle turbinate hypertrophy and polypoidal mucosa were seen equally by both.

As revealed from the Table IV associated lesions like concha bullosa and paradoxical middle turbinate were equally evident in $30 \%$ and $16.6 \%$ cases respectively by both endoscopy and CT scan.

\begin{tabular}{|c|c|c|c|}
\hline Sl. No. & Range (In years) & No. of Patients & Percentage \\
\hline 1. & $10-20$ & 6 & $10 \%$ \\
\hline 2. & $21-30$ & 16 & $26.6 \%$ \\
\hline 3. & $31-40$ & 20 & $33.3 \%$ \\
\hline 4. & $41-50$ & 6 & $10 \%$ \\
\hline 5. & $51-60$ & 4 & $6.6 \%$ \\
\hline 6. & $61-70$ & 8 & $13.3 \%$ \\
\hline \multicolumn{4}{|c|}{ Table I. Age Distribution } \\
\hline
\end{tabular}




\begin{tabular}{|c|c|c|c|c|c|c|c|}
\hline Symptom & $\begin{array}{c}\text { Nasal } \\
\text { Obstruction }\end{array}$ & Sneezing & Rhinorrhoea & Headache & Facial Pain & $\begin{array}{c}\text { Smell } \\
\text { Disturbances }\end{array}$ & $\begin{array}{c}\text { Post Nasal } \\
\text { Drip }\end{array}$ \\
\hline $\begin{array}{c}\text { Patients Complaints } \\
\text { with \% }\end{array}$ & $50 / 60$ & $42 / 60$ & $44 / 60$ & $34 / 60$ & $12 / 60$ & $22 / 60$ & $12 / 60$ \\
{$[83.3 \%)$} & $(70 \%)$ & {$[73.3 \%]$} & {$[56.6 \%]$} & {$[20 \%]$} & {$[36.6 \%]$} & {$[20 \%]$} \\
\hline \multicolumn{7}{|c|}{ Table II. Presenting Symptoms } \\
\hline
\end{tabular}

\begin{tabular}{|c|c|c|}
\hline Sinuses & $\begin{array}{c}\text { Evidence of } \\
\text { Involvement on } \\
\text { CT Scan } \\
\end{array}$ & \begin{tabular}{|c|} 
Evidence of \\
Involvement on \\
Endoscopy
\end{tabular} \\
\hline Maxillary (\%) & $40(66.6 \%)$ & $40(66.6 \%)$ \\
\hline Anterior Ethmoid (\%) & $44(73.3 \%)$ & $44(73.3 \%)$ \\
\hline Posterior Ethmoid (\%) & $18(30 \%)$ & $18(30 \%)$ \\
\hline Frontal (\%) & $14(23.3 \%)$ & $14(23.3 \%)$ \\
\hline Sphenoid (\%) & $10(16.6 \%)$ & $10(16.6 \%)$ \\
\hline \multicolumn{3}{|c|}{$\begin{array}{c}\text { Table III. CT and Endoscopic } \\
\text { Evaluation of Sinus Involvement }\end{array}$} \\
\hline
\end{tabular}

P value not applicable as both values are same.

\begin{tabular}{|c|c|c|}
\hline Associated Lesions & $\begin{array}{c}\text { Evident on CT } \\
\text { Scan }\end{array}$ & $\begin{array}{c}\text { Evident on Nasal } \\
\text { Endoscopy }\end{array}$ \\
\hline Concha Bullosa & $18(30 \%)$ & $18(30 \%)$ \\
\hline $\begin{array}{c}\text { Paradoxical Middle } \\
\text { Turbinate }\end{array}$ & $10(16.6 \%)$ & $10(16.6 \%)$ \\
\hline \multicolumn{2}{|c|}{ Table IV. Evaluation of Associated Lesions } \\
\hline
\end{tabular}

$\mathrm{P}$ value not applicable as both values are same.

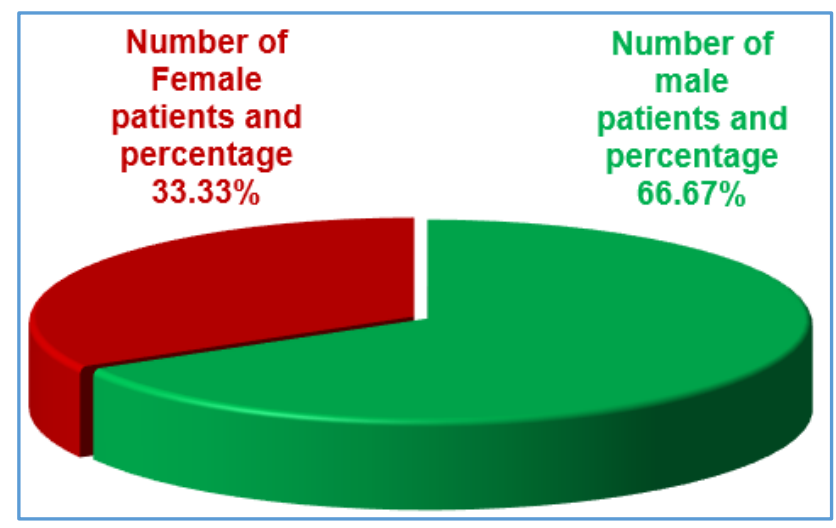

Figure 1. Distribution of Sex in Inflammatory Sinonasal Disease

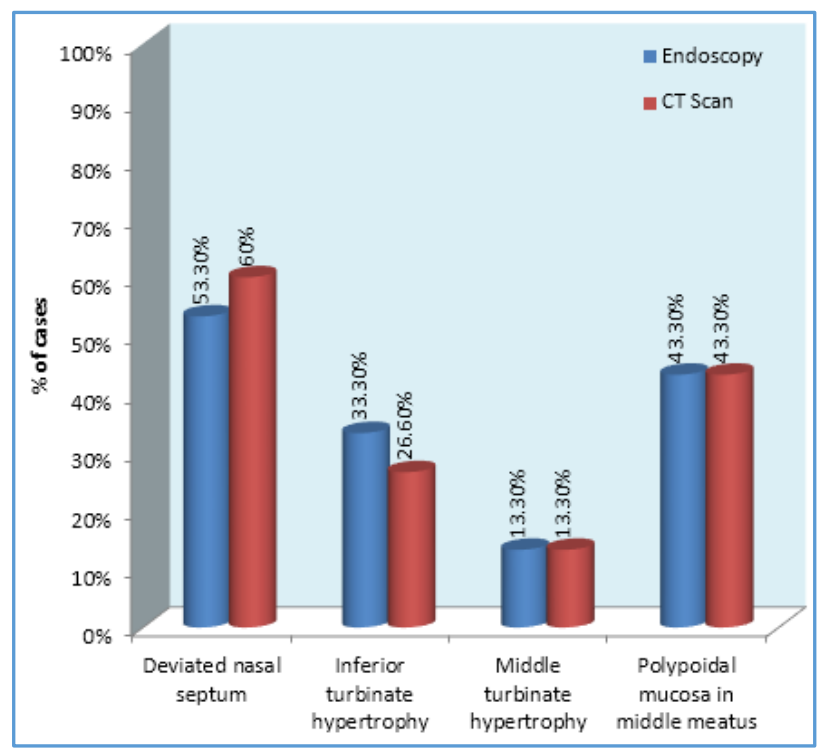

Figure 2. Finding seen on Endoscopy and CT-Scan $p$ value for Figure 2 findings is as under. ( $p>0.05$ ).

DNS: 0.60 (not significant).

Inferior turbinate hypertrophy: 0.57 (not significant).

Middle turbinate hypertrophy: (not applicable).

Polypoidal mucosa in middle meatus: (not applicable).

\section{DISCUSSION}

The chronic inflammatory sinonasal disease is a common clinical condition involving the nasal cavities and paranasal sinuses simultaneously.

The appraisal of this disease in past has been vague depending only on signs and symptoms, illumination tests in the dark and conventional plain skiagrams of paranasal sinuses as observed by McAlister et al (1989), who emphasised that it was impossible to visualise the ostiomeatal area and anatomic variants on plain sinus x-ray.

The real eye view with magnification on monitor by nasal endoscopies, introduced by Messerklinger (1967), (1978), Hopkins (1951) and Karl Storz (1966) enhanced all the anatomic and pathologic appearances of chronic rhinosinusitis as seen on CT scan PNS. The CT and endoscopy has made real understanding of key area ostiomeatal complex in CRS pathology.

The routine anterior and posterior rhinoscopy gives very little information as we can see only the structures which are visible to us. A thorough endoscopic examination of nose and paranasal sinuses is strongly recommended and if required CT scan can be done as both are complementary methods for diagnosis of patients with chronic rhinosinusitis.

Initially the diagnostic utility of nasal endoscopy in chronic rhinosinusitis in relation to CT scan had been assessed in few studies, but now the definite role of endoscopy has been well established for correct diagnosis of patients with symptoms of rhinosinusitis and evaluation of the factors that play a key role in treatment of this common disease. Anatomical structures like septal deviations, concha bullosa, paradoxical middle turbinate, middle meatus area may be evaluated by nasal endoscopic examination, without need for scanning methods. Therefore, endoscopic examination performed prior to CT scan is thought to be very useful and our results in study indicate there was almost perfect to substantial level of agreement between two methods.

In the present study, CRS had high preponderance in males $(66.6 \%)$ and is commonly encountered in the age group (10-70 years) with maximum no. of patients in third and fourth decade (59.9\%).

In the study conducted by Kirtane et al (1996), ${ }^{15,16}$ there were $61.5 \%$ males affected by the disease.

In our study, most common symptomatology was nasal obstruction $(83.3 \%)$ followed by rhinorrhoea, sneezing and headache.

In the study conducted by Kirtane et.al,15,16 the common complaint was nasal discharge followed by nasal obstructions. 
In our study, deviated nasal septum was a common clinical finding both with the endoscope (53.3\%) and CT scan $(60 \%)$ followed by middle meatus polypoidal mucosa $(43.3 \%)$ by both modalities. These findings suggest that both the modalities are univocal in diagnosing deviated nasal septum and middle meatus mucosa. Previous studies by Bolger et al 1991,99 Shroff 1996, Zinreich 1993,8 also showed the clinical finding of DNS to be more common in patients of chronic rhinosinusitis.

CT and endoscopic evaluation of the sinuses showed 73.3\% involvement of anterior ethmoids followed by maxillary sinus (66.6\%), posterior ethmoids $30 \%$, frontal sinus $23.3 \%$ and sphenoid sinus in $16.6 \%$.

Bolger et al[9,17] reported anterior ethmoids $84.3 \%$, maxillary sinus $77.7 \%$, post ethmoid in $38.6 \%$, frontal $36.6 \%$ and sphenoid in $25.4 \%$ to be involved with disease.

In the present study, concha bullosa was identified equally by nasal endoscopy and CT scan (30\%) cases with similar observation by Zinriech $(1993)^{8} 36 \%$, Shroff et al (1996) 33\%, somewhat less by Lloyd (1990) and little higher incidence at $53 \%$ by Bolger et al (1991). ${ }^{9}$

Another lesion observed equally by endoscopy and CT scan was paradoxical middle turbinate (16.6\%). Similar observations were seen by Lloyd (1990) 15\%, Zinreich (1993)8 15\% and Shroff et al (1996) 16\%.

Most of the patients showed turbinate hypertrophy by endoscopy examination compared to same affection on CT scan (13\%). In the current study, middle turbinate hypertrophy was seen equally by both modalities $(13.3 \%)$ and inferior turbinate hypertrophy $33 \%$ by endoscopy and $26.6 \%$ by CT scan. ${ }^{77]}$

\section{CONCLUSION}

Nasal endoscopy and CT scan are both good tools in diagnosing chronic rhinosinusitis and complementary to each other considering the results we obtained, but endoscopy being an outpatient procedure with unparalleled vision and brilliant illuminations of nose and paranasal sinuses is a better technique for detecting pathology of CRS as well as anatomical variations and should be preferred as an early diagnostic tool in clinical evaluation of disease. Diagnostic nasal endoscopy helps reduce CT utilisation, thereby reducing cost and radiation exposure in large population evaluated for CRS.

CT scan should be reserved for the patients with poor endoscopic visualisation, to define anatomical limits and patients who remain symptomatic despite medical management or those requiring surgical management for CRS.

\section{REFERENCES}

[1] Benninger MS, Ferguson BJ, Hadley JA, et al. Adult chronic rhinosinusitis: definitions, diagnosis, epidemiology and pathophysiology. Otolaryngol head neck Surg 2003;129(3 Suppl):S1-32.
[2] Fokkens W, Lund V, Mullol J. European position paper on rhinosinusitis and nasal polyps 2007. Rhinol suppl 2007;20:1-136.

[3] Lund VJ, Kennedy DW. Quantification for staging sinusitis. The staging and therapy group. Ann Otol Rhinol Laryngol Suppl 1995;167:17-21.

[4] Kennedy DW, Zinreich SJ, Rosenbaum AE, et al. Functional endoscopic sinus surgery. Theory and diagnostic evaluation. Arch Otolaryngol 1985;111(9):576-82.

[5] Lanza DC, Kennedy DW. Adult rhinosinusitis defined. Otolaryngol Head Neck Surg 1997;117(3 Pt 2):S1-7.

[6] Bailey BJ, Calhoun KH, Simpson CB, et al. CT evaluation of the paranasal sinuses in symptomatic and asymptomatic populations. Otolaryngol head neck Surg 1991;104(4):480-3.

[7] Duarte AF, de Soler RC, Zavarezzi F. Nasal endoscopy associated with paranasal sinus computerized tomography scan in the diagnosis of chronic nasal obstruction. Rev Bras Otorrinolaringol 2005;71(3):361-3.

[8] Zinreich J. Imaging of inflammatory sinus disease. Otolaryngol clin of North Am 1993;26(4):535-47.

[9] Bolger WE, Butzin CA, Parsons DS. Paranasal sinus bony anatomic variations and mucosal abnormalities: CT analysis for endoscopic sinus surgery. Laryngoscope 1991;101(1 Pt 1):56-64.

[10] Stankiewicz JA, Chow JM. Nasal endoscopy and the definition and diagnosis of chronic rhinosinusitis. Otolaryngol head neck surg 2002;126(6):623-7.

[11] Zinreich SJ, Kennedy DW, Rosenbaum AE, et al. Paranasal sinuses: CT imaging requirements for endoscopic surgery. Radiology 1987;163(3):769-75.

[12] Stammberger H, Wolf G. Headaches and sinus disease: the endoscopic approach. Ann Otol Rhinol Laryngol suppl 1988;134:3-23.

[13] Stammberger H. Endoscopic endonasal surgery-concepts in treatment of recurring rhinosinusitis. Part1. Anatomic and pathophysiologic considerations. Otolaryngol head neck surg 1986;94(2):143-7.

[14] Stammberger H, Philadelphia BC, Dekker P. The messerklinger technique. Functional endoscopic surgery 1991;62.

[15] Nayak SR, Desai PV, Kirtane MV, et al. Functional endoscopic sinuses surgery. A preliminary study. Indian J Otolaryngol 1991;43(3):126-9.

[16] Kirtane MV, Navani SB, Shetty PG, et al. Coronal screening sinus CT in inflammatory sinonasal disease. Ind J Radiol Image 1996;6(1):3-17.

[17] Bolger WE. Anatomy of the paranasal sinuses. In: Kennedy DW, Bolger WE, Zinreich J. Diseases of the sinuses: diagnosis and management 2001. 